\title{
Weighing in on Adipogenesis
}

\author{
Elizabeth R. Nunn, Abhijit B. Shinde and Elma Zaganjor* \\ Department of Molecular Physiology and Biophysics, Vanderbilt University School of Medicine, Nashville, TN, United States
}

\section{OPEN ACCESS}

Edited by:

Prashant Rajbhandari,

Icahn School of Medicine at Mount

Sinai, United States

Reviewed by:

Liang Guo,

Shanghai University of Sport, China

Jacqueline M. Stephens,

Louisiana State University,

United States

*Correspondence:

Elma Zaganjor

elma.zaganjor@vanderbilt.edu

Specialty section:

This article was submitted to

Metabolic Physiology,

a section of the journal

Frontiers in Physiology

Received: 24 November 2021

Accepted: 20 January 2022

Published: 25 February 2022

Citation:

Nunn $E R$, Shinde $A B$ and Zaganjor $E$ (2022) Weighing in on

Adipogenesis.

Front. Physiol. 13:821278.

doi: 10.3389/fphys.2022.821278
Obesity is a growing health concern worldwide because of its contribution to metabolic syndrome, type II diabetes, insulin resistance $(\mathrm{IR})$, and numerous cancers. In obesity, white adipose tissue (WAT) expands through two mechanisms: increase in adipocyte cell number by precursor cell differentiation through the process of adipogenesis (hyperplasia) and increase in existing mature adipocyte cell size (hypertrophy). While hypertrophy is associated with the negative effects of obesity on metabolic health, such as inflammation and lipotoxicity, adipogenesis prevents obesity-mediated metabolic decline. Moreover, in metabolically healthy obesity adipogenesis is increased. Thus, it is vital to understand the mechanistic basis for adipose expansion to inform novel therapeutic approaches to mitigate the dysfunction of this tissue and associated diseases. In this mini-review, we summarize recent studies on the regulation of adipogenesis and provide a perspective on targeting adipogenesis as a potential therapeutic avenue for metabolic disorders.

Keywords: epigenetics, mitochondria, metabolism, adipose, adipogenesis

\section{INTRODUCTION}

Although adiposity is linked to numerous diseases, adipocytes are critical for maintaining healthy systemic metabolism. Adipocytes perform important functions: coordinating energy balance, releasing nutrients in times of fasting, and storing nutrients in times of surplus. WAT is an endocrine organ that controls appetite, fertility, body temperature, glucose homeostasis, and insulin sensitivity by secreting hormones (adipokines), cytokines, and lipids. Adipose serves physical functions, such as cushioning organs like the heart, kidneys, ovaries, and extremities that experience high levels of stress, like the palms and heels. WAT expands through hyperplasia, an increase in adipocyte cell number through precursor cell differentiation via a process termed adipogenesis, and hypertrophy, an increase in existing, mature adipocyte cell size. Hypertrophy is accompanied by inflammation, lipotoxicity, and ectopic lipid accumulation, which may lead to IR. In contrast, adipogenesis prevents obesity-mediated metabolic decline. Genetically engineered mouse models which exhibit increased adipogenesis in the subcutaneous adipose depot are metabolically healthy despite increased adiposity (Kim et al., 2007; Kusminski et al., 2012). Therefore, inducing adipogenesis and limiting hypertrophy could present a therapeutic strategy for combating metabolic diseases induced by overnutrition. What are the molecular mechanisms that promote adipogenesis? Various signaling pathways and transcriptional and epigenetic regulators of adipogenesis have been elucidated. However, how cellular metabolism mechanistically regulates adipogenesis is only now being evaluated. In this mini-review, we summarize the literature on the role of cellular metabolism in adipogenesis and discuss the potential implications for the treatment of obesity-mediated disorders. 


\section{SIGNALING PATHWAYS IN ADIPOGENESIS}

Hormones and ligands regulate adipogenesis by modulating signaling pathways. Insulin engages the insulin receptor, activating the signaling cascade consisting of insulin receptor substrate (IRS), phosphoinositide 3-kinase (PI3K), AKT, and the mechanistic target of rapamycin complex 1 (mTORC1) to promote glucose uptake into differentiating preadipocytes. Glucocorticoids bind glucocorticoid receptor (GCR) to stimulate transcription via CCAAT/enhancer-binding proteins (C/EBPs) and peroxisome proliferator-activated receptor $\gamma$ (PPAR $\gamma$ ), enhancing insulin signaling required for adipogenesis (Ambele et al., 2020). The bone morphogenic proteins (BMPs), specifically BMP2 and BMP4, promote adipogenesis in part through SMAD4-mediated upregulation of PPAR $\gamma$ (Zamani and Brown, 2011). The hedgehog signaling pathway opposes adipogenesis through interference of BMP signaling (Suh et al., 2006; Garten et al., 2012). Canonical Wnt signaling suppresses adipogenesis through the stabilization of $\beta$-catenin that represses the PPAR $\gamma$-C/ EBP $\alpha$ complex (Kanazawa et al., 2005; Rosen and MacDougald, 2006). Other pathways, such as TGF $\beta$, FGF, ERK/MAPK, p38/ MAPK, and Notch signaling, can be pro- or anti-adipogenic depending on the experimental system, specific ligands used, and cell type, as well as the developmental or differentiation stage (Bost et al., 2005; Aouadi et al., 2006; Rosen and MacDougald, 2006; Zamani and Brown, 2011).

\section{TRANSCRIPTIONAL CONTROL OF ADIPOGENESIS}

PPAR $\gamma$ is one of the key transcriptional regulators of adipogenesis. Although early mechanistic studies investigating the function of PPAR $\gamma$ in adipogenesis were performed using in vitro model systems (Tontonoz et al., 1994; Rosen et al., 1999), the loss of WAT in PPAR $\gamma$-deficient mouse models such as chimeric PPAR $\gamma$-null (Rosen et al., 1999), tetraploid rescued (Barak et al., 1999), and adipocyte-specific PPAR $\gamma$ knockouts (KOs) (Wang et al., 2013a) provide evidence for its role in adipogenesis in vivo. The action of PPAR $\gamma$ promotes the transcriptional activity of another adipogenic regulator, co-activator CCAAT/ enhancer-binding protein- $\alpha(\mathrm{C} / \mathrm{EBP} \alpha)$ (Wu et al., 1999). When expressed ectopically, $\mathrm{C} / \mathrm{EBP} \alpha$ induces adipogenesis in fibroblasts (Freytag et al., 1994). However, C/EBP $\alpha$-deficient fibroblasts can only undergo partial adipogenesis, displaying several defects, such as decreased lipid accumulation and IR (Wu et al., 1999). The terminal step of embryonic adipogenesis is independent of $\mathrm{C} / \mathrm{EBP} \alpha$, possibly due to redundancy with other C/EBP family members, whereas it is essential for various WAT adipogenic conditions during adulthood (Wang et al., 2015). Moreover, $\operatorname{PPAR} \gamma$ can induce adipogenesis in the absence of $\mathrm{C} / \mathrm{EBP} \alpha$, but $\mathrm{C} / \mathrm{EBP} \alpha$ fails to do so in PPAR $\gamma$-deficient fibroblasts (Rosen et al., 2002). These observations suggest that C/EBP $\alpha$ and PPAR $\gamma$ work cooperatively in a single pathway, although PPAR $\gamma$ plays a central effector role in adipogenesis. C/EBP $\beta$ and
C/EBPS are expressed earlier during induction of the adipogenic program in 3T3-L1 cells, and they promote the expression of $\mathrm{C} / \mathrm{EBP} \alpha$ during the final phase of adipogenesis (Cao et al., 1991; Yeh et al., 1995). Additionally, mice lacking both C/ EBP $\beta$ and C/EBP $\delta$ showed a dramatic volume reduction of epidydimal WAT (eWAT) (Tanaka et al., 1997). Apart from these main factors, several other transcriptional regulators of adipogenesis have been identified and are reviewed in-depth elsewhere (Farmer, 2006; Rosen and Spiegelman, 2014; Lee et al., 2019).

\section{METABOLIC REGULATION OF ADIPOGENESIS}

Given that adipocytes are a reservoir for lipid stores and sense nutrient state to regulate organismal energy balance, metabolites themselves are significant regulators of adipogenesis. Glucose availability dictates adipocyte maturation, such that GLUT4mediated glucose uptake promotes adipogenesis through a number of distinct mechanisms (Kahn and Flier, 2000). First, glucose contributes to nicotinamide adenine dinucleotide phosphate (NADPH) synthesis through the pentose phosphate pathway. As NADPH is a required cofactor for lipogenesis, glucose directly mediates adipocyte differentiation through this pathway (Jackson et al., 2017). Another functional use of glucose is in acetyl-coenzyme A (acetyl-CoA) production. Preadipocytes derive acetyl-CoA for de novo lipogenesis from glucose, with the rate-limiting step in this pathway being catalyzed by pyruvate dehydrogenase (PDH). Upon differentiation, adipocytes decrease glucose contribution to lipogenic acetyl-CoA. To supplement this carbon pool, the catabolism of branched-chain amino acids (BCAAs), leucine, isoleucine, and valine, is another source of lipogenic acetyl-CoA that promotes adipogenesis (Green et al., 2016). Mitochondrial BCAA catabolism involves transamination of BCAAs by the branched-chain amino transferase (BCAT), generating branched-chain $\alpha$-keto acids (BCKAs). BCKAs are oxidized by the BCAA dehydrogenase (BCKDH) complex. During the remainder of oxidation BCAA carbons are either lost as $\mathrm{CO}_{2}$ or contribute carbons to the tricarboxylic acid (TCA) cycle as succinyl-CoA or acetyl-CoA (Neinast et al., 2019a). In adipogenesis, a mitochondrial sirtuin, SIRT4, promotes leucine catabolism by increasing the activity of methylcrotonyl-CoA carboxylase (MCCC1), an enzyme that catalyzes the carboxylation of 3-methylcrotonyl-CoA to 3-methylglutaconyl-CoA (Anderson et al., 2017; Zaganjor et al., 2021). The induction of leucine catabolism occurs early in the process of differentiation and further promotes PPAR $\gamma$ function (Zaganjor et al., 2021). Adipogenesis reduces the contribution of glutamine carbon to fatty acids and instead promotes de novo glutamine synthesis (Green et al., 2016). Moreover, knockdown of glutaminase (Gls), which promotes glutaminolysis by deamination of glutamine to glutamate, stimulates adipogenic fate in skeletal stem cells (Yu et al., 2019). Therefore, glutamine oxidation appears inhibitory to adipogenesis, although the mechanism remains elusive. Because almost all the aforementioned nutrient oxidation pathways, other than the pentose phosphate pathway, occur in the mitochondria, 
these studies demonstrate a vital contribution of mitochondrial function in adipogenesis (Figure 1).

\section{MITOCHONDRIAL FUNCTION IN ADIPOCYTE DIFFERENTIATION}

Adipogenesis involves a 20 - to 30 -fold increase in the concentration of mitochondrial proteins as determined by both proteomics and electron microscopy (Wilson-Fritch et al., 2003). Mechanistically, the transcriptional program consisting of cyclic adenosine monophosphate (CAMP) responsive element binding protein (CREB), PPAR $\gamma, \mathrm{C} / \mathrm{EBP} \alpha$, estrogen-related receptor $\alpha$ $(\mathrm{ERR} \alpha)$, and PPAR $\gamma$ co-activator 1 (PGC-1) are engaged to support mitochondrial biogenesis. Mitochondrial respiratory capacity increases with differentiation, suggesting that mitochondria are biochemically altered (Wilson-Fritch et al., 2003). Successful adipogenesis relies on mitochondrial biogenesis.

Which mitochondrial functions support differentiation? Beyond nutrient oxidation within the mitochondria to generate acetyl-CoA, the mitochondrial electron transport chain (ETC) is a source of reactive oxygen species (ROS) that can also modulate differentiation. ROS can regulate mitochondrial dynamics, increasing mitochondrial fission in bone marrow mesenchymal stem cells (BMSCs) to promote adipogenesis (Guo et al., 2020). While mitochondrialtargeted antioxidants block adipogenesis (Tormos et al., 2011), constitutively high levels of ROS have an inhibitory effect on adipogenesis through the induction of a transcriptional repressor C/EBP homologous protein 10 (CHOP-10) (Carriere et al., 2004; Furukawa et al., 2004). The toxic elevation of ROS also causes structural cellular damage to mitochondrial proteins, lipids, and DNA, including damage to the ETC, which results in depletion of ATP and nicotinamide adenine dinucleotide (NAD) (Rolo et al., 2012). In human clinical trials, antioxidants were not successful in preventing risks of type II diabetes (T2D), possibly because of the beneficial effects of ROS on adipogenesis (Castro et al., 2016). Gaining insight into the threshold of ROS that has beneficial, pro-adipogenic effects may be critical in treating metabolic dysfunction.

\section{EPIGENETIC CONTROL OF ADIPOGENESIS}

Epigenetic modifications including acetylation, methylation, phosphorylation, and ribosylation are regulated by several

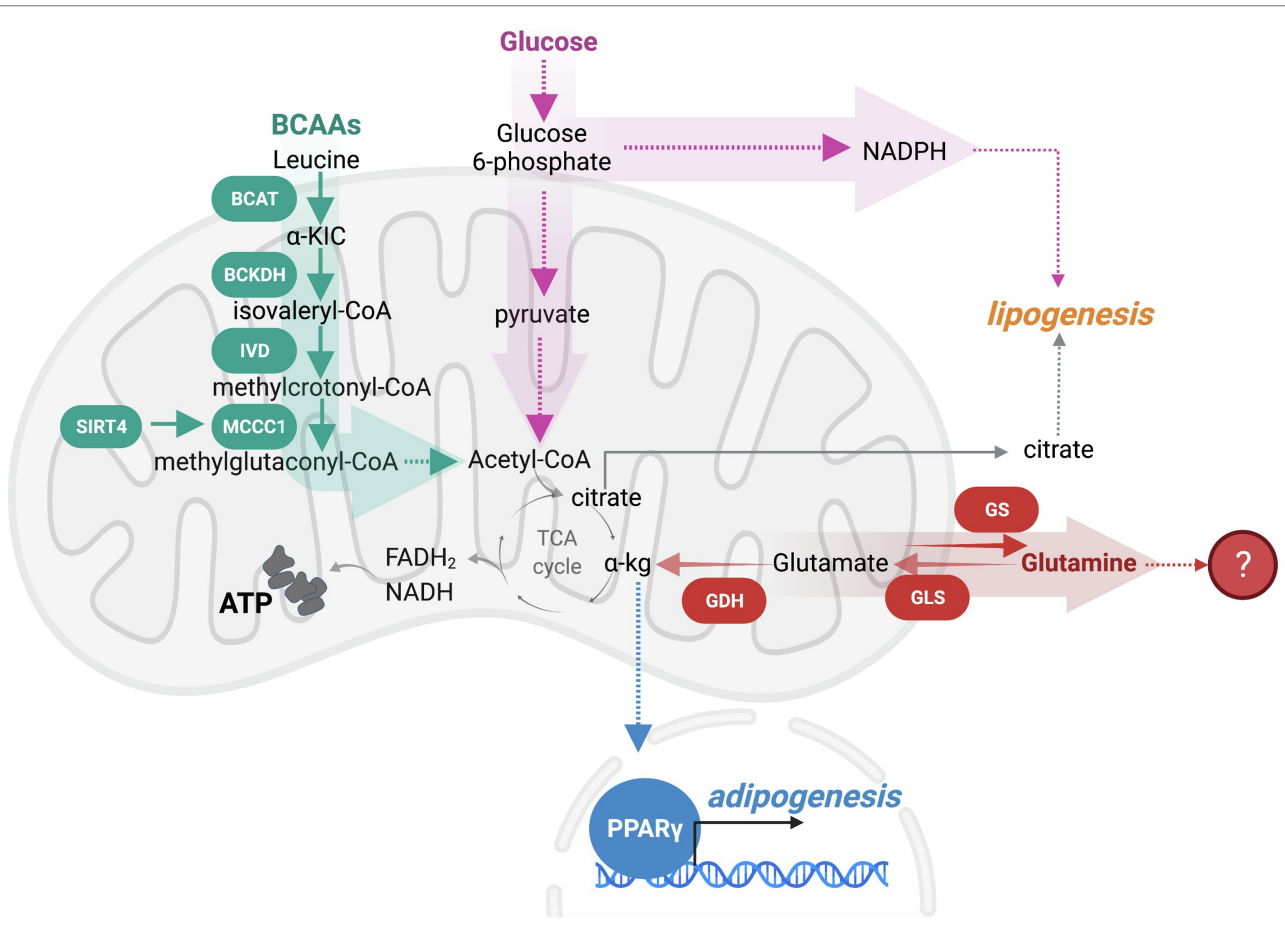

FIGURE 1 | Nutrient oxidation in the mitochondria modulates adipogenesis. Branched-chain amino acids (BCAAs) and glucose catabolism within the mitochondria produce ATP and metabolic intermediates that promote adipogenesis. As such, leucine catabolism is an early regulator of adipogenesis that is initiated by a BCAA transaminase (BCAT) generating $\alpha$-ketoisocaproic acid (a-KIC). a-KIC is irreversibly oxidized by the BCAA dehydrogenase (BCKDH) complex forming isovaleryl-CoA. Isovaleryl-CoA dehydrogenase (IVD) converts isovaleryl-CoA to methylcrotonyl-CoA. Sirtuin SIRT4 induction of methylcrotonyl-CoA carboxylase (MCCC1) promotes the carboxylation of 3-methylcrotonyl-CoA to 3-methylglutaconyl-CoA, which can be further oxidized to produce acetyl-CoA. BCAA catabolism promotes the PPAR $y$-mediated transcriptional adipogenic program. While a portion of glucose is oxidized to generate acetyl-CoA, some glucose is diverted away from the mitochondrial oxidation to the pentose phosphate pathway (PPP) to support the production of nicotinamide adenine dinucleotide phosphate (NADPH), a required cofactor for lipogenesis. Glutaminolysis appears to oppose adipogenesis, although the mechanistic understanding of fuel switching that supports adipogenesis is limited. 
different classes of enzymes that connect the energetic status of the cell to changes in the epigenetic landscape. Epigenetic regulators may have positive or negative effects on adipogenesis and include histone acetyltransferases (HATs) and deacetylases (HDACs), histone, and DNA methyltransferases (HMTs and DNMTs), and demethylases that include ten-eleven translocation DNA demethylases (TETs), and histone demethylases, such as JmjC domain-containing histone demethylases (JMJDs) and histone lysine demethylase 1 (LSD1) (Musri et al., 2010; Chatterjee et al., 2011; Lee and Ge, 2014) (Figure 2). The epigenetic landscape is complex and multi-faceted, as mono-, di-, and tri-methylation can occur at both histone and DNA methylation marks, and acetylation, phosphorylation, and ribosylation are just a few of the dynamic modifications that can be added and removed by enzymatic regulators (Lee et al., 2019). Epigenetic patterns are altered as preadipocytes commit to this lineage and differentiate into mature adipocytes,

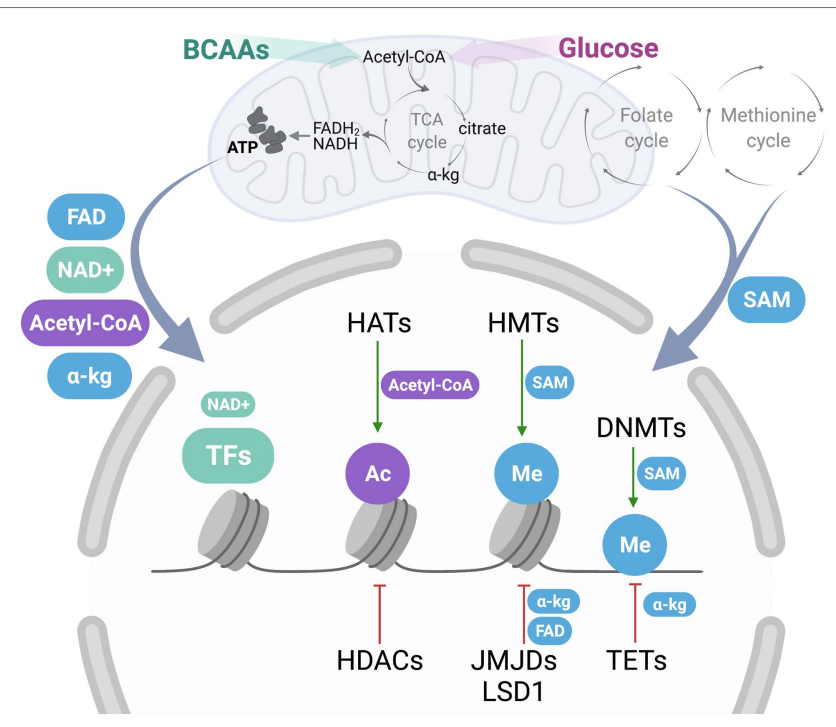

FIGURE 2 | Communication between mitochondria-derived metabolites and nuclear epigenetic regulators. One-carbon metabolism, consisting of folate and methionine cycle, generates S-adenosylmethionine (SAM), a metabolite contributing to epigenetic regulation. Oxidation of fuels, such as BCAAs and glucose, coupled to the activity of the tricarboxylic acid (TCA) cycle and the electron transport chain (ETC) produce important metabolic byproducts, including flavin adenine dinucleotide (FAD), nicotinamide adenine dinucleotide (NAD+), acetyl-coenzyme A (acetyl-CoA), and $\alpha$-ketoglutarate (a-kg). Once exported out of the mitochondria, these metabolites may contribute to cytosolic signaling, or act as cofactors and donor groups to epigenetic regulators in the nucleus, including histone acetyltransferases (HATs) and deacetylases (HDACs), histone and DNA methyltransferases (HMTs and DNMTs), and demethylases that include ten-eleven translocation DNA demethylases (TETs), and histone demethylases, such as JmjC domaincontaining histone demethylases (JMJDs) and histone lysine demethylase 1 (LSD1), as well as other nuclear transcription factors (TFs). HATs add acetyl groups (Ac, in purple) to histones using acetyl-CoA as a substrate, while HDACs remove these groups. HMTs utilize SAM as a donor of a methyl group (Me, in blue) to histones, while methyl groups are removed by JMJDs, which utilize a-kg, or LSD1, which couples with FAD synthesis. Similarly, DMNTs utilize SAM for DNA methylation, while a-kg is a cofactor used by TETs for DNA demethylation. Additionally, NAD+ acts as an important cofactor for nuclear TFs and other regulatory enzymes, including PARP-1 and sirtuins. and these changes can silence or promote the expression of particular genes as necessary. For example, histone H3K9 di-methylation via G9a in the promoter region of Ppary represses its expression and subsequently blocks differentiation (Wang et al., 2013b). LSD1 decreases H3K9 di-methylation while maintaining $\mathrm{H} 3 \mathrm{~K} 4$ di-methylation at the Cebpa promoter to promote adipogenesis (Musri et al., 2010). LSD1 can also demethylate H3K9 to support adipogenesis through PPAR $\gamma$ activity (Jang et al., 2017). Furthermore, a specific bivalent chromatin signature $\mathrm{H} 3 \mathrm{~K} 4 / \mathrm{H} 3 \mathrm{~K} 9 \mathrm{me} 3$ keeps the expression of Ppary and Cebpa at low levels, allowing preadipocytes to remain primed for differentiation (Matsumura et al., 2015). This complex array of histone and DNA modifications that are added and removed by epigenetic regulators rely on metabolites generated within the mitochondria.

\section{METABOLIC REGULATION OF EPIGENETICS IN ADIPOGENESIS}

Metabolic intermediates and cofactors, including acetyl-CoA, $\alpha$-ketoglutarate, and $\mathrm{NAD}+$, are derived in the mitochondria through nutrient oxidation or the TCA cycle, where they can then act as cofactors to mitochondrial enzymes or be exported into the cytosol. The TCA cycle produces metabolites that contribute to epigenetic modification (Figure 2). For example, mutations to the TCA cycle enzyme isocitrate dehydrogenase (IDH) and production of 2 -hydroxyglutarate (2HG) decrease $\alpha$-ketoglutarate levels. The loss of $\alpha$-ketoglutarate contributes to the decreased activity of lysine-specific demethylase 4C (KDM4C), causing an increase in $\mathrm{H} 3 \mathrm{~K} 9$ methylation that inhibits adipogenesis ( $\mathrm{Lu}$ et al., 2012). In the following sections, we will discuss research that has illuminated how important metabolites contribute to epigenetic regulation in adipogenesis.

\section{Acetyl-CoA}

Acetyl-CoA is a component of central carbon metabolism that provides carbon for lipogenesis. Beyond this role, acetyl-CoA is a donor group for histone acetylation via the activities of HATs (Shi and Tu, 2015). Within the mitochondria, acetyl-CoA is generated through $\beta$-oxidation of fatty acids, from pyruvate, and via branched-chain amino acid catabolism, and is reported to exit mitochondria through several mechanisms, including via the carnitine/acylcarnitine translocase and via SLC25A1 as citrate (Madiraju et al., 2009; Zaidi et al., 2012). Within the cytosol, the activity of ATP-citrate lyase (ACLY) converts citrate to acetyl-CoA and oxaloacetate. Cytosolic acetyl-CoA can take part in lipogenesis, steroidogenesis, or become a building block for glutamine, proline, and arginine (Pietrocola et al., 2015). Acetyl-CoA can also be regenerated from citrate in the nucleus by ACLY, driving a global increase in histone acetylation in 3 T3-L1 cells (Wellen et al., 2009). When acetyl-CoA is depleted following siRNA-mediated silencing of ACLY or following glucose restriction, histone acetylation levels are decreased, 
and adipocyte differentiation is inhibited (Wellen et al., 2009). However, some compensation has been observed as the loss of ACLY stimulates the expression of acyl-CoA synthetase short-chain family member 2 (ACSS2), which can generate acetyl-CoA from acetate (Zhao et al., 2016). High-fat diet leads to a decrease in whole-tissue acetyl-CoA levels in murine WAT and a subsequent decrease in H3K23ac, showing a correlation between diet, acetyl-CoA levels, and histone acetylation (Carrer et al., 2017). Elucidating the influence of diet on metabolite levels and histone alterations could have important implications for understanding obesity and associated diseases.

\section{NAD+}

Metabolic pathways including the TCA cycle, ETC, fatty acid oxidation, and glycolysis influence the redox state of the cell through NAD+ metabolism. NAD+ is a small molecule that can be synthesized from precursors including nicotinamide (NAM), nicotinic acid (NA), and nicotinamide riboside (NR); through the de novo synthesis pathway from tryptophan; and through NAD+ salvage (Katsyuba et al., 2020). NAD+ acts as both a cofactor in redox reactions and a substrate for enzymes like poly [ADP-ribose] polymerases (PARPs) and sirtuins, which act as deacylases in the mitochondria, nucleus, and cytoplasm (Katsyuba et al., 2020). NAD+ is thought to exist in distinct mitochondrial, cytosolic, and nuclear compartments due to the presence of distinct nicotinamide mononucleotide adenylyltransferase (NMNAT) isoforms in these cellular locations (Berger et al., 2005; Nikiforov et al., 2011). NAD+ usage in these compartments causes fluctuations in nicotinamide mononucleotide (NMN) and NAD+ availability that have differing effects on epigenetic events that influence adipogenesis. Within the mitochondrial compartment, increased NAD+ production via increased flux through the TCA cycle is required to induce adipogenesis in 3T3-L1 cells (Okabe et al., 2020). Induction of adipogenesis increases the expression of cytosolic NMNAT2 resulting in elevated cytosolic NAD+ (Ryu et al., 2018). This activity of NMNAT2 decreases the availability of NMN for nuclear NMNAT1 and leads to a decline in nuclear NAD+. As nuclear NAD+ drops, PARP-1 activity decreases, leading to a subsequent reduction in its ADP-ribosylation of CEBPa. Loss of this modification increases the CEBPa proadipogenic transcriptional program (Ryu et al., 2018). Furthermore, NMNAT1 activity and synthesis of NAD+ direct PARP-1 ability to PARylate aspartate and glutamate residues on histones (Huang et al., 2020). These findings illustrate the temporal and compartment-specific influence of $\mathrm{NAD}+$ metabolism on the cellular epigenetic state that modulates adipogenesis.

Compartment-specific alterations in NAD+ levels may also impact sirtuin activity. Increased mitochondrial NAD+ may promote the activity of SIRT4, an inducer of adipogenesis, while decreased nuclear NAD+ may decrease the activity of SIRT1, a negative regulator of adipogenesis (Mayoral et al., 2015; Zaganjor et al., 2021). NAD+ compartmentalization and availability may fine-tune adipogenesis via multiple independent mechanisms. SLC25A51 has been recently identified as a mitochondrial NAD+ transporter (Girardi et al., 2020; Kory et al., 2020; Luongo et al., 2020), which may present an opportunity to modulate NAD+ compartmentalization and cell differentiation.

\section{SAM, FAD, and $\alpha$-Ketoglutarate}

Other metabolites have been shown or suggested to influence epigenetic regulation, but their roles are not as well-defined. One such metabolite is S-adenosylmethionine (SAM). SAM acts as a major methyl donor and is utilized by histone lysine methyltransferases (HKMTs) as a cofactor, where the donation of a methyl group yields S-adenosylhomocysteine (SAH) (Wiese and Bannister, 2020). This metabolite is derived by S-adenosyl methionine transferase (MAT) by the catabolism of methionine and ATP or one-carbon metabolism (Wiese and Bannister, 2020). The addition of SAM to culture media induces differentiation in 3T3-L1 cells, although the precise mechanism has not been elucidated (Liu et al., 2013).

Another metabolite that participates in epigenetic regulation is flavin adenine dinucleotide (FAD). FAD is a cofactor utilized in redox reactions by demethylases, such as LSD1 and, once reduced, can be re-oxidized by molecular oxygen (Berger and Sassone-Corsi, 2016). Through coupling with FAD synthesis, LSD1 epigenetically regulates the expression of energy expenditure genes through the removal of mono- and di-methylation of H3K4, and knockdown of LSD1 results in activation of mitochondrial respiration and lipolysis in mature adipocytes (Hino et al., 2012). FAD levels increase both in differentiating 3T3-L1 preadipocytes as well as in mature 3T3-L1 cells following palmitate exposure to stimulate lipid storage and suppress PGC-1 $\alpha$ (Hino et al., 2012). Alternatively, the knockdown of FAD-dependent LSD1 in differentiating 3T3-L1 cells leads to a significant decrease in lipid accumulation and expression of adipogenic regulator Cebpa (Musri et al., 2010). These findings suggest that FAD-dependent enzymes directly impact epigenetic regulation in adipocytes, indicating that FAD itself is a significant metabolite modulating adipogenesis.

Similarly, $\alpha$-ketoglutarate acts as a cofactor for histone demethylases, as well as for DNA demethylation by TETs (Carey et al., 2015). Okabe et al. report that an increase in $\alpha$-ketoglutarate is likely responsible for demethylation of $\mathrm{H} 3 \mathrm{~K} 9 \mathrm{me} 3$ at the Ppary promoter, supporting adipogenesis (Okabe et al., 2020). These studies support the model that metabolites relay nutrient state information to alter gene expression and cell fate.

\section{THERAPEUTIC INTERVENTION}

Adipogenesis is important in systemic metabolic health and increasing adipogenesis may profoundly improve outcomes for patients with obesity-related diseases, such as diabetes. Mutations in genes that regulate lipid droplet formation lead to lipodystrophy, a condition characterized by severe IR and dyslipidemia (Magre et al., 2001; Agarwal et al., 2002; Ghaben and Scherer, 2019). Inhibition of adipogenesis through the depletion of PPAR $\gamma$ in the progenitor population results in pathological WAT expansion in mice (Shao et al., 2018). 
Collectively, these studies suggest the protective effects of adipogenesis and warrant the search for therapeutic interventions that promote the process. Thiazolidinediones (TZDs), synthetic PPAR $\gamma$ activators, although promising in their ability to promote adipogenesis, adipose tissue beiging, insulin sensitivity and reduced inflammation, also carry reported cardiac and osteoporosis risks (Ahmadian et al., 2013). Evaluating metabolic targets in their ability to induce adipogenesis in the context of diet-induced obesity may have therapeutic benefits. Notably, BCAAs accumulate in diabetic patients and BCAA catabolism in adipose tissue is dysfunctional in diabetic mouse models (Felig et al., 1969; Newgard et al., 2009; Neinast et al., 2019b). Identifying small molecules that potentiate this pathway may improve adipogenesis and overall health. Similarly, high-fat diet downregulates ACLY in WAT, alters histone acetylation, and disrupts lipid storage (Carrer et al., 2017). Future studies are necessary to evaluate the impact of maintaining ACLY levels on adipogenesis and metabolic health after a dietary challenge. Recent technological advances in mass spectrometry, compartmentalized metabolomics and general growth of the field will allow for discoveries on new regulators of adipose development (Chen et al., 2017; Collins et al., 2021). Elucidating

\section{REFERENCES}

Agarwal, A. K., Arioglu, E., De Almeida, S., Akkoc, N., Taylor, S. I., Bowcock, A. M., et al. (2002). AGPAT2 is mutated in congenital generalized lipodystrophy linked to chromosome 9q34. Nat. Genet. 31, 21-23. doi: 10.1038/ng880

Ahmadian, M., Suh, J. M., Hah, N., Liddle, C., Atkins, A. R., Downes, M., et al. (2013). PPARgamma signaling and metabolism: the good, the bad and the future. Nat. Med. 19,557-566. doi: 10.1038/nm.3159

Ambele, M. A., Dhanraj, P., Giles, R., and Pepper, M. S. (2020). Adipogenesis: a complex interplay of multiple molecular determinants and pathways. Int. J. Mol. Sci. 21:4283. doi: $10.3390 / \mathrm{ijms} 21124283$

Anderson, K. A., Huynh, F. K., Fisher-Wellman, K., Stuart, J. D., Peterson, B. S., Douros, J. D., et al. (2017). SIRT4 is a lysine deacylase that controls leucine metabolism and insulin secretion. Cell Metab. 25, 838-855.e815. doi: 10.1016/j. cmet.2017.03.003

Aouadi, M., Laurent, K., Prot, M., Le Marchand-Brustel, Y., Binétruy, B., and Bost, F. (2006). Inhibition of p38MAPK increases adipogenesis from embryonic to adult stages. Diabetes 55, 281-289. doi: 10.2337/diabetes.55.02. 06.db05-0963

Barak, Y., Nelson, M. C., Ong, E. S., Jones, Y. Z., Ruiz-Lozano, P., Chien, K. R., et al. (1999). PPARgamma is required for placental, cardiac, and adipose tissue development. Mol. Cell 4, 585-595. doi: 10.1016/S1097-2765(00)80209-9

Berger, F., Lau, C., Dahlmann, M., and Ziegler, M. (2005). Subcellular compartmentation and differential catalytic properties of the three human nicotinamide mononucleotide adenylyltransferase isoforms. J. Biol. Chem. 280, 36334-36341. doi: 10.1074/jbc.M508660200

Berger, S. L., and Sassone-Corsi, P. (2016). Metabolic signaling to chromatin. Cold Spring Harb. Perspect. Biol. 8:a019463. doi: 10.1101/cshperspect.a019463

Bost, F., Aouadi, M., Caron, L., and Binétruy, B. (2005). The role of MAPKs in adipocyte differentiation and obesity. Biochimie 87, 51-56. doi: 10.1016/j. biochi.2004.10.018

Cao, Z., Umek, R. M., and McKnight, S. L. (1991). Regulated expression of three C/EBP isoforms during adipose conversion of 3T3-L1 cells. Genes Dev. 5, 1538-1552. doi: 10.1101/gad.5.9.1538

Carey, B. W., Finley, L. W., Cross, J. R., Allis, C. D., and Thompson, C. B. (2015). Intracellular alpha-ketoglutarate maintains the pluripotency of embryonic stem cells. Nature 518, 413-416. doi: 10.1038/nature13981

Carrer, A., Parris, J. L. D., Trefely, S., Henry, R. A., Montgomery, D. C., Torres, A., et al. (2017). Impact of a high-fat diet on tissue acyl-CoA and the metabolic drivers of adipogenesis may lead to novel approaches to mitigate obesity-mediated disorders.

\section{AUTHOR CONTRIBUTIONS}

All authors listed have made a substantial, direct, and intellectual contribution to the work and approved it for publication.

\section{FUNDING}

EZ was supported by the Vanderbilt Digestive Diseases Research Center Grant (P30 058404).

\section{ACKNOWLEDGMENTS}

We apologize to the investigators whose work we were unable to highlight due to space constraints. We thank our colleague Jessica Spinelli for the helpful discussion on the manuscript. Figures were created with BioRender.com.

histone acetylation levels. J. Biol. Chem. 292, 3312-3322. doi: 10.1074/jbc. M116.750620

Carriere, A., Carmona, M. C., Fernandez, Y., Rigoulet, M., Wenger, R. H., Penicaud, L., et al. (2004). Mitochondrial reactive oxygen species control the transcription factor CHOP-10/GADD153 and adipocyte differentiation: a mechanism for hypoxia-dependent effect. J. Biol. Chem. 279, 40462-40469. doi: 10.1074/jbc.M407258200

Castro, J. P., Grune, T., and Speckmann, B. (2016). The two faces of reactive oxygen species (ROS) in adipocyte function and dysfunction. Biol. Chem. 397, 709-724. doi: 10.1515/hsz-2015-0305

Chatterjee, T. K., Idelman, G., Blanco, V., Blomkalns, A. L., Piegore, M. G. Jr., Weintraub, D. S., et al. (2011). Histone deacetylase 9 is a negative regulator of adipogenic differentiation. J. Biol. Chem. 286, 27836-27847. doi: $10.1074 /$ jbc.M111.262964

Chen, W. W., Freinkman, E., and Sabatini, D. M. (2017). Rapid immunopurification of mitochondria for metabolite profiling and absolute quantification of matrix metabolites. Nat. Protoc. 12, 2215-2231. doi: 10.1038/nprot.2017.104

Collins, S. L., Koo, I., Peters, J. M., Smith, P. B., and Patterson, A. D. (2021). Current challenges and recent developments in mass spectrometry-based metabolomics. Annu Rev Anal Chem. (Palo Alto, Calif) 14, 467-487. doi: 10.1146/annurev-anchem-091620-015205

Farmer, S. R. (2006). Transcriptional control of adipocyte formation. Cell Metab. 4, 263-273. doi: 10.1016/j.cmet.2006.07.001

Felig, P., Marliss, E., and Cahill, G. F. Jr. (1969). Plasma amino acid levels and insulin secretion in obesity. N. Engl. J. Med. 281, 811-816. doi: 10.1056/ NEJM196910092811503

Freytag, S. O., Paielli, D. L., and Gilbert, J. D. (1994). Ectopic expression of the CCAAT/enhancer-binding protein alpha promotes the adipogenic program in a variety of mouse fibroblastic cells. Genes Dev. 8, 1654-1663. doi: 10.1101/ gad.8.14.1654

Furukawa, S., Fujita, T., Shimabukuro, M., Iwaki, M., Yamada, Y., Nakajima, Y., et al. (2004). Increased oxidative stress in obesity and its impact on metabolic syndrome. J. Clin. Invest. 114, 1752-1761. doi: 10.1172/JCI21625

Garten, A., Schuster, S., and Kiess, W. (2012). The insulin-like growth factors in adipogenesis and obesity. Endocrinol. Metab. Clin. N. Am. 41, 283-295. doi: $10.1016 /$ j.ecl.2012.04.011

Ghaben, A. L., and Scherer, P. E. (2019). Adipogenesis and metabolic health. Nat. Rev. Mol. Cell Biol. 20, 242-258. doi: 10.1038/s41580-018-0093-Z 
Girardi, E., Agrimi, G., Goldmann, U., Fiume, G., Lindinger, S., Sedlyarov, V., et al. (2020). Epistasis-driven identification of SLC25A51 as a regulator of human mitochondrial NAD import. Nat. Commun. 11:6145. doi: 10.1038/ s41467-020-19871-x

Green, C. R., Wallace, M., Divakaruni, A. S., Phillips, S. A., Murphy, A. N., Ciaraldi, T. P., et al. (2016). Branched-chain amino acid catabolism fuels adipocyte differentiation and lipogenesis. Nat. Chem. Biol. 12, 15-21. doi: 10.1038/nchembio.1961

Guo, J., Ren, R., Yao, X., Ye, Y., Sun, K., Lin, J., et al. (2020). PKM2 suppresses osteogenesis and facilitates adipogenesis by regulating beta-catenin signaling and mitochondrial fusion and fission. Aging (Albany NY) 12, 3976-3992. doi: 10.18632/aging.102866

Hino, S., Sakamoto, A., Nagaoka, K., Anan, K., Wang, Y., Mimasu, S., et al. (2012). FAD-dependent lysine-specific demethylase-1 regulates cellular energy expenditure. Nat. Commun. 3:758. doi: 10.1038/ncomms1755

Huang, D., Camacho, C. V., Setlem, R., Ryu, K. W., Parameswaran, B., Gupta, R. K., et al. (2020). Functional interplay between histone H2B ADP-ribosylation and phosphorylation controls adipogenesis. Mol. Cell 79, 934-949.e914. doi: 10.1016/j.molcel.2020.08.002

Jackson, R. M., Griesel, B. A., Gurley, J. M., Szweda, L. I., and Olson, A. L. (2017). Glucose availability controls adipogenesis in mouse 3T3-L1 adipocytes via up-regulation of nicotinamide metabolism. J. Biol. Chem. 292, 18556-18564. doi: $10.1074 /$ jbc.M117.791970

Jang, M. J., Park, U. H., Kim, J. W., Choi, H., Um, S. J., and Kim, E. J. (2017). CACUL1 reciprocally regulates SIRT1 and LSD1 to repress PPARgamma and inhibit adipogenesis. Cell Death Dis. 8:3201. doi: 10.1038/s41419-017-0070-z

Kahn, B. B., and Flier, J. S. (2000). Obesity and insulin resistance. J. Clin. Invest. 106, 473-481. doi: 10.1172/JCI10842

Kanazawa, A., Tsukada, S., Kamiyama, M., Yanagimoto, T., Nakajima, M., and Maeda, S. (2005). Wnt5b partially inhibits canonical Wnt/beta-catenin signaling pathway and promotes adipogenesis in 3T3-L1 preadipocytes. Biochem. Biophys. Res. Commun. 330, 505-510. doi: 10.1016/j.bbrc.2005.03.007

Katsyuba, E., Romani, M., Hofer, D., and Auwerx, J. (2020). NAD(+) homeostasis in health and disease. Nat. Metab. 2, 9-31. doi: 10.1038/s42255-019-0161-5

Kim, J. Y., van de Wall, E., Laplante, M., Azzara, A., Trujillo, M. E., Hofmann, S. M., et al. (2007). Obesity-associated improvements in metabolic profile through expansion of adipose tissue. J. Clin. Invest. 117, 2621-2637. doi: 10.1172/ JCI31021

Kory, N., Uit de Bos, J., van der Rijt, S., Jankovic, N., Gura, M., Arp, N., et al. (2020). MCART1/SLC25A51 is required for mitochondrial NAD transport. Sci. Adv. 6:eabe5310. doi: 10.1126/sciadv.abe5310

Kusminski, C. M., Holland, W. L., Sun, K., Park, J., Spurgin, S. B., Lin, Y., et al. (2012). MitoNEET-driven alterations in adipocyte mitochondrial activity reveal a crucial adaptive process that preserves insulin sensitivity in obesity. Nat. Med. 18, 1539-1549. doi: 10.1038/nm.2899

Lee, J. E., and Ge, K. (2014). Transcriptional and epigenetic regulation of PPARgamma expression during adipogenesis. Cell Biosci. 4:29. doi: 10.1186/2045-3701-4-29

Lee, J. E., Schmidt, H., Lai, B., and Ge, K. (2019). Transcriptional and epigenomic regulation of adipogenesis. Mol. Cell. Biol. 39, e00601-e00618. doi: 10.1128/ MCB.00601-18

Liu, Y., Lv, W., Yu, B., Ju, T., Yang, F., Jiang, M., et al. (2013). S-adenosylmethionineinduced adipogenesis is accompanied by suppression of Wnt/beta-catenin and Hedgehog signaling pathways. Mol. Cell. Biochem. 382, 59-73. doi: 10.1007/s11010-013-1718-3

Lu, C., Ward, P. S., Kapoor, G. S., Rohle, D., Turcan, S., Abdel-Wahab, O., et al. (2012). IDH mutation impairs histone demethylation and results in a block to cell differentiation. Nature 483, 474-478. doi: 10.1038/ nature 10860

Luongo, T. S., Eller, J. M., Lu, M. J., Niere, M., Raith, F., Perry, C., et al. (2020). SLC25A51 is a mammalian mitochondrial $\mathrm{NAD}(+)$ transporter. Nature 588, 174-179. doi: 10.1038/s41586-020-2741-7

Madiraju, P., Pande, S. V., Prentki, M., and Madiraju, S. R. (2009). Mitochondrial acetylcarnitine provides acetyl groups for nuclear histone acetylation. Epigenetics 4, 399-403. doi: 10.4161/epi.4.6.9767

Magre, J., Delepine, M., Khallouf, E., Gedde-Dahl, T. Jr., Van Maldergem, L., Sobel, E., et al. (2001). Identification of the gene altered in Berardinelli-Seip congenital lipodystrophy on chromosome 11q13. Nat. Genet. 28, 365-370. doi: $10.1038 / n g 585$
Matsumura, Y., Nakaki, R., Inagaki, T., Yoshida, A., Kano, Y., Kimura, H., et al. (2015). H3K4/H3K9me3 bivalent chromatin domains targeted by lineage-specific DNA methylation pauses adipocyte differentiation. Mol. Cell 60, 584-596. doi: 10.1016/j.molcel.2015.10.025

Mayoral, R., Osborn, O., McNelis, J., Johnson, A. M., Oh, D. Y., Izquierdo, C. L., et al. (2015). Adipocyte SIRT1 knockout promotes PPARgamma activity, adipogenesis and insulin sensitivity in chronic-HFD and obesity. Mol. Metab. 4, 378-391. doi: 10.1016/j.molmet.2015.02.007

Musri, M. M., Carmona, M. C., Hanzu, F. A., Kaliman, P., Gomis, R., and Parrizas, M. (2010). Histone demethylase LSD1 regulates adipogenesis. J. Biol. Chem. 285, 30034-30041. doi: 10.1074/jbc.M110.151209

Neinast, M. D., Jang, C., Hui, S., Murashige, D. S., Chu, Q., Morscher, R. J., et al. (2019b). Quantitative analysis of the whole-body metabolic fate of branched-chain amino acids. Cell Metab. 29, 417-429.e414. doi: 10.1016/j. cmet.2018.10.013

Neinast, M., Murashige, D., and Arany, Z. (2019a). Branched chain amino acids. Annu. Rev. Physiol. 81, 139-164. doi: 10.1146/annurev-physiol020518-114455

Newgard, C. B., An, J., Bain, J. R., Muehlbauer, M. J., Stevens, R. D., Lien, L. F., et al. (2009). A branched-chain amino acid-related metabolic signature that differentiates obese and lean humans and contributes to insulin resistance. Cell Metab. 9, 311-326. doi: 10.1016/j.cmet.2009.02.002

Nikiforov, A., Dolle, C., Niere, M., and Ziegler, M. (2011). Pathways and subcellular compartmentation of NAD biosynthesis in human cells: from entry of extracellular precursors to mitochondrial NAD generation. J. Biol. Chem. 286, 21767-21778. doi: 10.1074/jbc.M110.213298

Okabe, K., Nawaz, A., Nishida, Y., Yaku, K., Usui, I., Tobe, K., et al. (2020). NAD+ metabolism regulates Preadipocyte differentiation by enhancing alphaketoglutarate-mediated histone $\mathrm{H} 3 \mathrm{~K} 9$ demethylation at the PPARgamma promoter. Front. Cell Dev. Biol. 8:586179. doi: 10.3389/fcell.2020.586179

Pietrocola, F., Galluzzi, L., Bravo-San Pedro, J. M., Madeo, F., and Kroemer, G. (2015). Acetyl coenzyme A: a central metabolite and second messenger Cell Metab. 21, 805-821. doi: 10.1016/j.cmet.2015.05.014

Rolo, A. P., Teodoro, J. S., and Palmeira, C. M. (2012). Role of oxidative stress in the pathogenesis of nonalcoholic steatohepatitis. Free Radic. Biol. Med. 52, 59-69. doi: 10.1016/j.freeradbiomed.2011.10.003

Rosen, E. D., Hsu, C. H., Wang, X., Sakai, S., Freeman, M. W., Gonzalez, F. J., et al. (2002). C/EBPalpha induces adipogenesis through PPARgamma: a unified pathway. Genes Dev. 16, 22-26. doi: 10.1101/gad.948702

Rosen, E. D., and MacDougald, O. A. (2006). Adipocyte differentiation from the inside out. Nat. Rev. Mol. Cell Biol. 7, 885-896. doi: 10.1038/nrm2066

Rosen, E. D., Sarraf, P., Troy, A. E., Bradwin, G., Moore, K., Milstone, D. S., et al. (1999). PPARgamma is required for the differentiation of adipose tissue in vivo and in vitro. Mol. Cell 4, 611-617. doi: 10.1016/S10972765(00)80211-7

Rosen, E. D., and Spiegelman, B. M. (2014). What we talk about when we talk about fat. Cell 156, 20-44. doi: 10.1016/j.cell.2013.12.012

Ryu, K. W., Nandu, T., Kim, J., Challa, S., DeBerardinis, R. J., and Kraus, W. L. (2018). Metabolic regulation of transcription through compartmentalized $\mathrm{NAD}(+)$ biosynthesis. Science 360:ean5780. doi: 10.1126/science.aan5780

Shao, M., Vishvanath, L., Busbuso, N. C., Hepler, C., Shan, B., Sharma, A. X., et al. (2018). De novo adipocyte differentiation from Pdgfrbeta(+) preadipocytes protects against pathologic visceral adipose expansion in obesity. Nat. Commun. 9:890. doi: 10.1038/s41467-018-03196-x

Shi, L., and Tu, B. P. (2015). Acetyl-CoA and the regulation of metabolism: mechanisms and consequences. Curr. Opin. Cell Biol. 33, 125-131. doi: 10.1016/j.ceb.2015.02.003

Suh, J. M., Gao, X., McKay, J., McKay, R., Salo, Z., and Graff, J. M. (2006). Hedgehog signaling plays a conserved role in inhibiting fat formation. Cell Metab. 3, 25-34. doi: 10.1016/j.cmet.2005.11.012

Tanaka, T., Yoshida, N., Kishimoto, T., and Akira, S. (1997). Defective adipocyte differentiation in mice lacking the C/EBPbeta and/or C/EBPdelta gene. EMBO J. 16, 7432-7443. doi: 10.1093/emboj/16.24.7432

Tontonoz, P., Hu, E., and Spiegelman, B. M. (1994). Stimulation of adipogenesis in fibroblasts by PPARgamma 2, a lipid-activated transcription factor. Cell 79, 1147-1156. doi: 10.1016/0092-8674(94)90006-X

Tormos, K. V., Anso, E., Hamanaka, R. B., Eisenbart, J., Joseph, J., Kalyanaraman, B., et al. (2011). Mitochondrial complex III ROS regulate adipocyte differentiation Cell Metab. 14, 537-544. doi: 10.1016/j.cmet.2011.08.007 
Wang, F., Mullican, S. E., DiSpirito, J. R., Peed, L. C., and Lazar, M. A. (2013a). Lipoatrophy and severe metabolic disturbance in mice with fat-specific deletion of PPARgamma. Proc. Natl. Acad. Sci. U. S. A. 110, 18656-18661. doi: $10.1073 /$ pnas. 1314863110

Wang, Q. A., Tao, C., Jiang, L., Shao, M., Ye, R., Zhu, Y., et al. (2015). Distinct regulatory mechanisms governing embryonic versus adult adipocyte maturation. Nat. Cell Biol. 17, 1099-1111. doi: 10.1038/ncb3217

Wang, L., Xu, S., Lee, J. E., Baldridge, A., Grullon, S., Peng, W., et al. (2013b). Histone H3K9 methyltransferase G9a represses PPARgamma expression and adipogenesis. EMBO J. 32, 45-59. doi: 10.1038/emboj.2012.306

Wellen, K. E., Hatzivassiliou, G., Sachdeva, U. M., Bui, T. V., Cross, J. R., and Thompson, C. B. (2009). ATP-citrate lyase links cellular metabolism to histone acetylation. Science 324, 1076-1080. doi: 10.1126/science.1164097

Wiese, M., and Bannister, A. J. (2020). Two genomes, one cell: mitochondrialnuclear coordination via epigenetic pathways. Mol. Metab. 38:100942. doi: 10.1016/j.molmet.2020.01.006

Wilson-Fritch, L., Burkart, A., Bell, G., Mendelson, K., Leszyk, J., Nicoloro, S., et al. (2003). Mitochondrial biogenesis and remodeling during adipogenesis and in response to the insulin sensitizer rosiglitazone. Mol. Cell. Biol. 23, 1085-1094. doi: 10.1128/MCB.23.3.1085-1094.2003

Wu, Z., Rosen, E. D., Brun, R., Hauser, S., Adelmant, G., Troy, A. E., et al. (1999). Cross-regulation of C/EBP alpha and PPARgamma controls the transcriptional pathway of adipogenesis and insulin sensitivity. Mol. Cell 3, 151-158. doi: 10.1016/S1097-2765(00)80306-8

Yeh, W. C., Cao, Z., Classon, M., and McKnight, S. L. (1995). Cascade regulation of terminal adipocyte differentiation by three members of the C/EBP family of leucine zipper proteins. Genes Dev. 9, 168-181. doi: 10.1101/gad.9.2.168

Yu, Y., Newman, H., Shen, L., Sharma, D., Hu, G., Mirando, A. J., et al. (2019). Glutamine metabolism regulates proliferation and lineage allocation in skeletal stem cells. Cell Metab. 29, 966-978.e964. doi: 10.1016/j.cmet.2019.01.016
Zaganjor, E., Yoon, H., Spinelli, J. B., Nunn, E. R., Laurent, G., Keskinidis, P., et al. (2021). SIRT4 is an early regulator of branched-chain amino acid catabolism that promotes adipogenesis. Cell Rep. 36:109345. doi: 10.1016/j. celrep.2021.109345

Zaidi, N., Swinnen, J. V., and Smans, K. (2012). ATP-citrate lyase: a key player in cancer metabolism. Cancer Res. 72, 3709-3714. doi: 10.1158/0008-5472. CAN-11-4112

Zamani, N., and Brown, C. W. (2011). Emerging roles for the transforming growth factor-\{beta\} superfamily in regulating adiposity and energy expenditure. Endocr. Rev. 32, 387-403. doi: 10.1210/er.2010-0018

Zhao, S., Torres, A., Henry, R. A., Trefely, S., Wallace, M., Lee, J. V., et al. (2016). ATP-citrate lyase controls a glucose-to-acetate metabolic switch. Cell Rep. 17, 1037-1052. doi: 10.1016/j.celrep.2016.09.069

Conflict of Interest: The authors declare that the research was conducted in the absence of any commercial or financial relationships that could be construed as a potential conflict of interest.

Publisher's Note: All claims expressed in this article are solely those of the authors and do not necessarily represent those of their affiliated organizations, or those of the publisher, the editors and the reviewers. Any product that may be evaluated in this article, or claim that may be made by its manufacturer, is not guaranteed or endorsed by the publisher.

Copyright (c) 2022 Nunn, Shinde and Zaganjor. This is an open-access article distributed under the terms of the Creative Commons Attribution License (CC BY). The use, distribution or reproduction in other forums is permitted, provided the original author(s) and the copyright owner(s) are credited and that the original publication in this journal is cited, in accordance with accepted academic practice. No use, distribution or reproduction is permitted which does not comply with these terms. 